\title{
CIRPASS: a NIR integral field and multi-object spectrograph
}

lan Robert Parry, Craig D. Mackay, Rachel A. Johnson, Richard G. McMahon, Andrew Dean, et al.

Ian Robert Parry, Craig D. Mackay, Rachel A. Johnson, Richard G. McMahon, Andrew Dean, Anamparambu N. Ramaprakash, David L. King, Jim M. Pritchard, Steve R. Medlen, Chris S. Sabbey, Richard S. Ellis, Alfonso Aragon-Salamanca, "CIRPASS: a NIR integral field and multi-object spectrograph," Proc. SPIE 4008, Optical and IR Telescope Instrumentation and Detectors, (16 August 2000); doi: 10.1117/12.395438 


\title{
CIRPASS: a NIR integral field and multi-object spectrograph
}

\author{
Ian Parry ${ }^{\mathrm{a}}$, Craig Mackay ${ }^{\mathrm{a}}$, Rachel Johnson ${ }^{\mathrm{a}}$, Richard McMahon ${ }^{\mathrm{a}}$, Andrew Dean ${ }^{\mathrm{a}}$, Anamparambu \\ Ramaprakash $^{\mathrm{a}}$, David King ${ }^{\mathrm{a}}$, Jim Pritchard ${ }^{\mathrm{a}}$, Steve Medlen ${ }^{\mathrm{a}}$, Chris Sabbey ${ }^{\mathrm{a}}$, Richard Ellis ${ }^{\mathrm{b}}$, Alfonso \\ Aragón-Salamanca ${ }^{c}$ \\ ${ }^{a}$ University of Cambridge, Institute of Astronomy, Madingley Rd, Cambridge, CB3 OHA, UK \\ ${ }^{\mathrm{b}}$ 105-24 Astronomy, Caltech, Pasadena, CA 91125, USA \\ ${ }^{\mathrm{c} S c h o o l}$ of Physics \& Astronomy, University of Nottingham, University Park, \\ Nottingham, NG7 2RD, UK
}

\begin{abstract}
We describe CIRPASS (the Cambridge Infra-Red PAnoramic Survey Spectrograph) which is currently being completed at the Institute of Astronomy in Cambridge. The optical design (which includes a hardware $\mathrm{OH}$ suppression capability) is presented and contrasted with more conventional software-suppression designs. The limiting magnitudes on Gemini are expected to be $\mathrm{J} \sim 22.6$ and $\mathrm{H} \sim 21.7$ in a 3 hour exposure.
\end{abstract}

Keywords: Infrared spectroscopy, OH suppression, Integral field spectroscopy, Multi-object spectroscopy.

\section{INTRODUCTION}

Our main objective in building CIRPASS ${ }^{1}$ is to provide a near-IR spectrograph for the study of extremely faint high redshift galaxies. Because of the need to obtain spatially resolved spectroscopy on individual galaxies and large samples of spectroscopy for survey work, CIRPASS has both an integral field mode and a multi-object mode. In both cases the spectrograph is fibre fed. Observations of this nature also require a very large telescope aperture and we will be using CIRPASS on the Gemini $8 \mathrm{~m}$ telescopes. To maximize wavelength coverage and minimize detector dark current and readnoise CIRPASS uses a HAWAII-II 2048×2048 array. To further minimize the background and improve sensitivity we use both software and hardware suppression to minimize the effects of the strong $\mathrm{OH}$ airglow and we minimize the thermal background from the instrument by a combination of cooling and using blocking filters.

CIRPASS is based on the design of the OH suppressor unit for COHSI (Cambridge OH Suppression Instrument ${ }^{2,3}$ ) and we have reused the large COHSI optics in its construction. COHSI used three dispersing elements to remove the OH lines and give a large wavelength coverage in one hit using two $256 \times 256$ PICNIC detectors. To do this three dispersing elements were used in the optical path. The first grating produced a high resolution spectrum on the suppression mask and the second then completely undispersed the light back to an OH-suppressed polychromatic slit image. This was then redispersed with a grism to give the correct resolution to match the small detectors. The option of partially undispersing with the second grating to directly give the required final resolution was not chosen because, to provide a cold slit and a cold stop, the whole suppressor has to be cooled. With the newer, larger $1024 \times 1024$ and $2048 \times 2048$ arrays it is not necessary to have a different spectral resolution at the mask and at the detector so it is possible to use a single grating and thereby achieve much higher throughput.

We are currently operating CIRPASS on the $40 \mathrm{~cm}$ Schmidt telescope in Cambridge. The test configuration uses a temporary camera which has twice the required focal length and is not cooled. The IFU is the 100-element one we built for COHSI ${ }^{5,6}$ and the detector is a HAWAII-I array. The fact that we can couple the IFU to a small classical Schmidt telescope strongly demonstrates how versatile and telescope-independent CIRPASS is. By matching the etendue of the telescope to that of CIRPASS we get a similar background sky to that at Gemini and we can therefore test CIRPASS very realistically before shipping to Hawaii. The final tests will use the new cryogenic camera and the new 499-element IFU. CIRPASS will be a visitor instrument at Gemini and will be operated by the Institute of Astronomy on behalf of the Gemini community. Initially it will be used on Gemini North and then later on Gemini South. It will be possible to use CIRPASS with the University of Hawaii's adaptive optics system, Hokupa' $a^{4}$. 


\section{SPECTROGRAPH OPTO-MECHANICAL DESIGN}

Key features of CIRPASS are:

- Integral Field Spectroscopy with 499 spatial samples and several image scale options (see Table 3)

- Multi-object spectroscopy with 128 fibres

- Can be used in conjunction with AO systems including Hokupa'a

- Operation in the range $0.85-1.8 \mu \mathrm{m}$

- Large wavelength coverage $(\Delta \lambda=0.44 \mu \mathrm{m})$ at $\mathrm{R} \sim 3100$ in a single integration with a 2048 array

- Spectral resolutions up to $\mathrm{R} 26,000$

- Throughput comparable to a conventional faint object spectrograph

- Limiting magnitudes of $\mathrm{J} 22.6$ and $\mathrm{H} 21.7$ in 3 hours (see Table 2)

\subsection{Spectrograph Optical Design}

The 3-D optical layout of the CIRPASS spectrograph is shown in Figure 1 and a side view showing the camera dewar and the refrigerated enclosure is shown in Figure 2. There are two fibre feeds: a plug-plate MOS feed and an integral field unit (IFU). Initially only the IFU feed will be available. The spectrograph will sit on the rotating observing floor of Gemini between the two sets of stairs that lead to the elevation axis. The design re-uses the large COHSI suppressor unit, which disperses the light, filters out the $\mathrm{OH}$ lines and delivers a collimated beam to the system's exit pupil. The suppressor unit has to be cooled to $-40^{\circ} \mathrm{C}$ for optimal operation longward of $1.4 \mu \mathrm{m}$. The final part of the system is a cryogenic camera which images the light on to a Rockwell HAWAII-I or HAWAII-II array. The optics in this camera are cooled to $\sim 68 \mathrm{~K}$ using liquid nitrogen below atmospheric pressure.

The first part of the suppressor system is an off-axis Schmidt in double-pass, the first pass acting as the collimator and the second acting as a camera. The fore-optics at the telescope end of the fibre feed are arranged so that FRD is taken into account, maximising the light collected by the $\mathrm{f} / 5.5$ collimator. The simplicity of the collimator arrangement will keep scattering to a minimum in this part of the system allowing the mask mirror to reject a very large fraction of the total $\mathrm{OH}$ light coming in from the sky.

For observations at the faintest limits, a reflection grating working in first order provides spectra on the mask at a spectral resolution which is high enough $(\mathrm{R} \sim 3100$ at $1.4 \mu \mathrm{m})$ to suppress the $\mathrm{OH}$ lines. The beam size at the grating is $150 \mathrm{~mm}$ in diameter allowing standard, large Richardson Labs gratings to be used. The $\mathrm{f} / 5.5$ spectrum at the mask is physically reduced to the size of the HAWAII array by a third pass through the Schmidt (which collimates the beam) and an f/1.35 camera. To select the central wavelength we tilt a beam steering mirror to view the appropriate mask area. Tilting the grating would also select the central wavelength but would misalign the $\mathrm{OH}$ spectrum with the mask.

We are currently using a grating that has $400 \mathrm{l} / \mathrm{mm}$ and is blazed at $1.2 \mu \mathrm{m}$. We will also provide 4 additional grating options. One will be another $400 \mathrm{l} / \mathrm{mm}$ grating blazed at $1.6 \mu \mathrm{m}$ which will give a factor of $1.3 \times$ more throughput in $\mathrm{H}$. For MOS work the larger fibres require twice the dispersion to achieve $\mathrm{R} 3000$ so we will use an $800 \mathrm{l} / \mathrm{mm}$ grating. The other two will be a $400 \mathrm{l} / \mathrm{mm}$ grating blazed at $4.65 \mu \mathrm{m}$ which gives $\mathrm{R} \sim 11,000$ in third order and an echelle to give R 26,000. The near-Littrow design which provides hardware suppression allows these high dispersion options whereas a conventional spectrograph design with a large camera-collimator angle would not.

We originally planned to use a 1024 array and a fully achromatic camera, which allowed the use of cross-dispersion but this was ruled out as too costly and risky. The camera design, which is now under construction, can feed a 2048 array with excellent image quality thereby doubling the wavelength range covered. The previous design used aspheric surfaces and troublesome materials such as Barium Fluoride and Calcium Fluoride. To make the new camera design cheaper and faster to produce our current design uses only regular Schott glasses and spherical surfaces. The number of elements has also been reduced. The design makes no attempt to correct chromatic aberration optically and we correct this by tilting the detector slightly with respect to the optical axis. The camera can use either a 1024 or a 2048 array. The slit length projects to 1024 pixels so the IFU field of view and the multiplex gain are the same whichever array is used. However, the HAWAII-II array gives twice the wavelength coverage. Ray-tracing the full design we find that $80 \%$ of the light from a point source at the slit ends up in one detector pixel for the worst case images. 


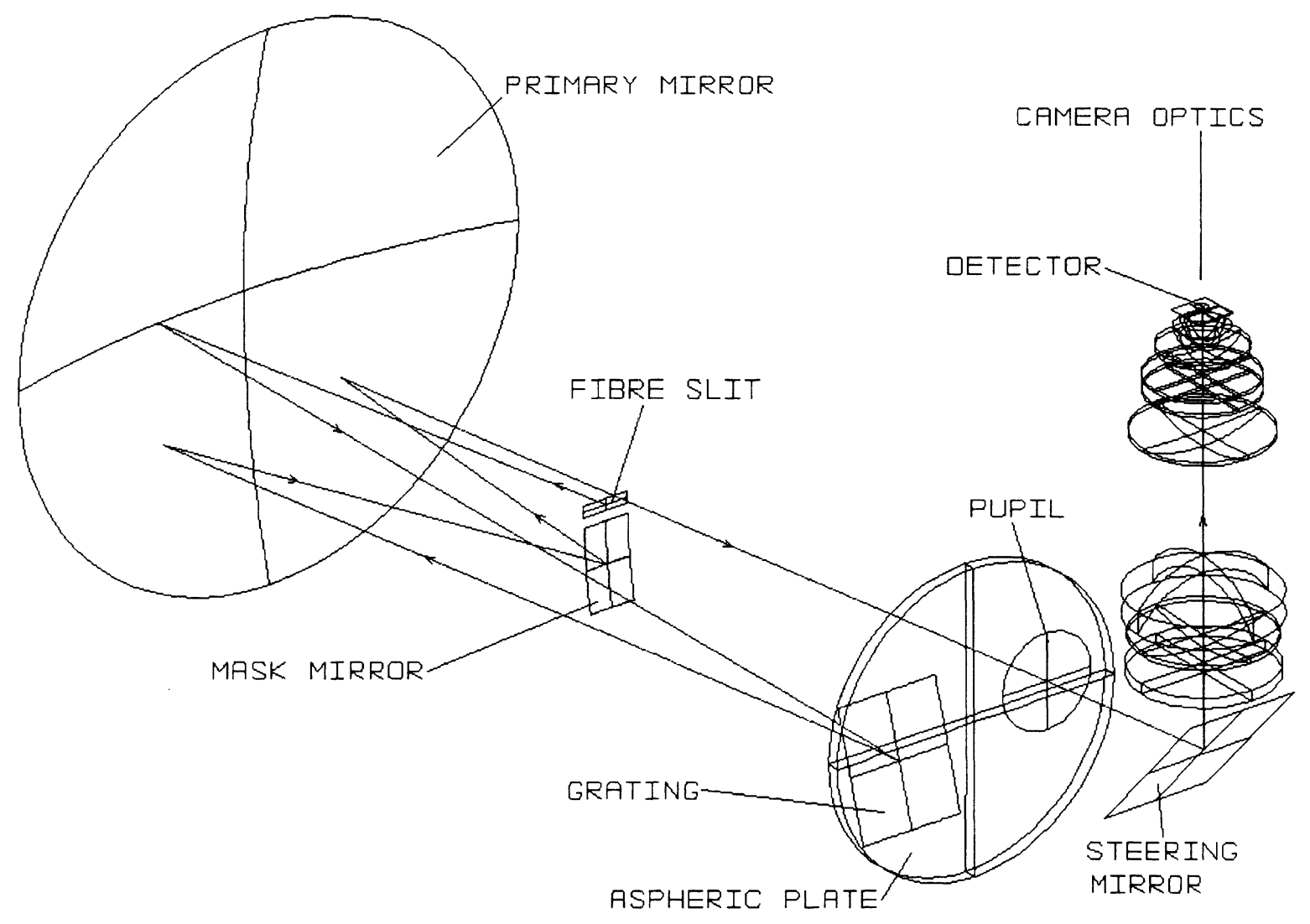

Figure 1. 3d view of the CIRPASS optics.

The chromatic aberrations of the camera rule out cross-dispersed spectra so for the MOS mode we will have 128 fibres with a single order per fibre. For 4 pixel resolution elements we get one quarter of the full $(0.85-1.8 \mu \mathrm{m})$ wavelength coverage in one shot on the 2048 array and to get full spectral coverage it will be necessary to scan through 4 steering mirror positions. This gives more full spectra per night than the echelle option we initially considered because what we lose in wavelength coverage we more than recover in multiplex gain.

New masks are needed for both IFU and MOS modes because they use different dispersions. The existing COHSI masks are not suitable because they are not wide enough to accommodate the full CIRPASS slit length. We also have a blank mask (a plain mirror) for the high dispersion modes that do not require hardware suppression.

\subsection{The cryogenic camera design}

Figure 3 shows the design of the cryogenic camera. The structure shown is $1024 \mathrm{~mm}$ from top to bottom. This is mounted rigidly on to the suppressor structure and the whole of the vacuum dewar is cooled along with the rest of the suppressor unit. The dewar window is therefore at $-40^{\circ} \mathrm{C}$. The internal optics and their mounts are cooled to $\sim 68 \mathrm{~K}$ by pumping on the liquid nitrogen can. The camera optics consist of 5 lenses which have spherical surfaces and are made of standard Schott glasses. The largest lens is $224 \mathrm{~mm}$ in diameter. Each lens is mounted in an aluminium ring that has splits cut in it so that when the lens and the mount are cooled to $68 \mathrm{~K}$ the ring can flex to accommodate the differential contraction of the two materials. This flexing is concentric with the optical axis and does not introduce any de-centres or tilts. The entire cold assembly including the nitrogen can, the detector mounts, the lens mounts and the filter wheel are made of aluminium to avoid differential contraction problems. The cold assembly is supported mainly by G10 trusses at the top of the dewar with some 
additional G10 supports around the filter wheel. These latter supports can flex to accommodate the differential contraction between the cold assembly and the dewar walls.

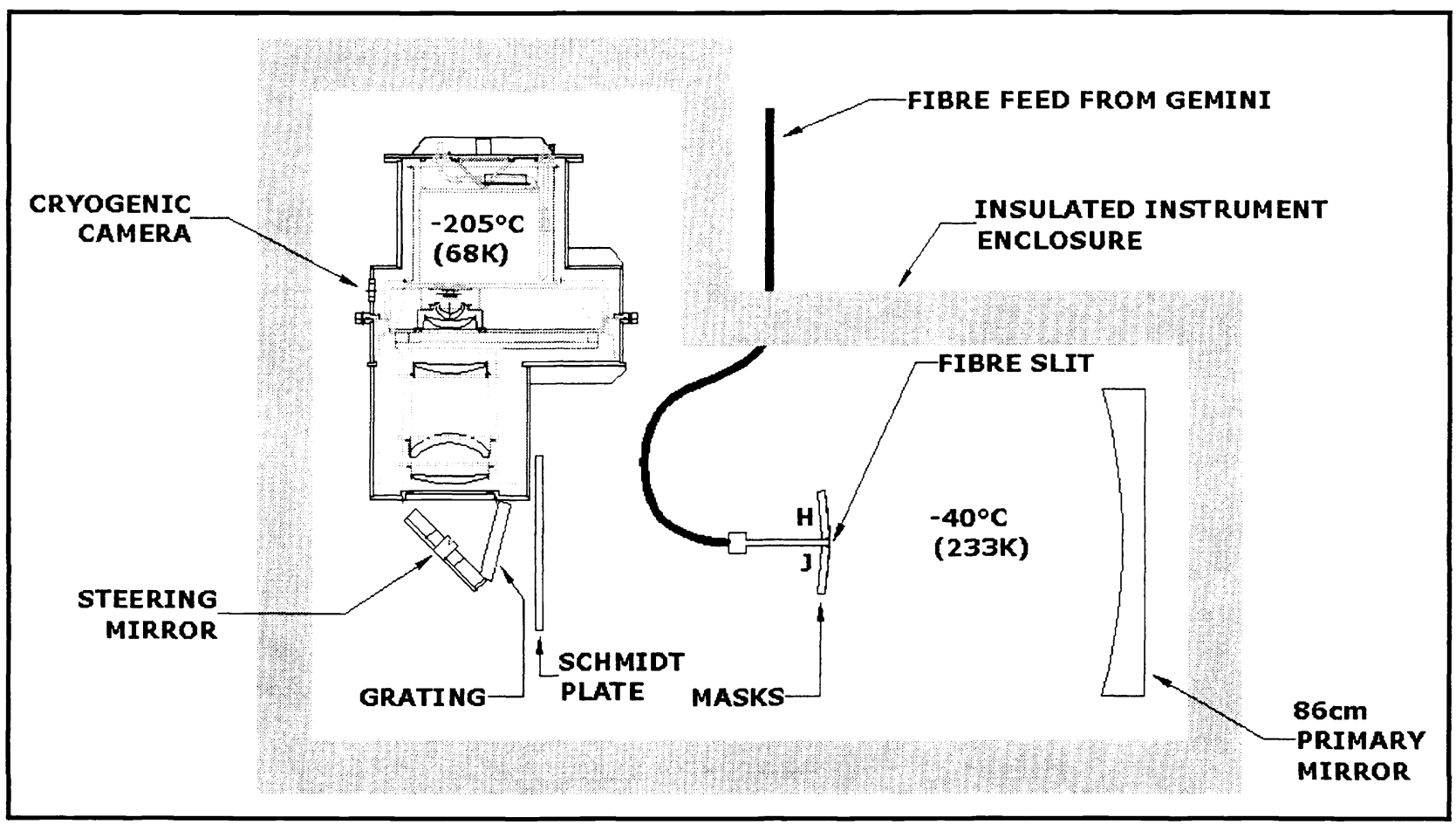

Figure 2. Schematic view of CIRPASS showing the instrument cooling scheme

The main vacuum vessel consists of three main parts: the top plate with the nitrogen can and its associated plumbing attached; the main body which has the electrical connectors; and the lower cover which includes the dewar window. Ease of assembly and disassembly from both a mechanical and an electrical point of view is an important feature of the design. The inner cold structure is hidden from the outer dewar walls by thin aluminium heat shields.

The detector module is mounted on an adjustable three point kinematic seating arrangement that has a stepper motor on each of the three mounting points. This allows the detector to be moved along the optical axis for focusing and allows it to be tilted with respect to the optical axis to accommodate the camera's change in focal length with wavelength. Positioning is by step counting and referencing to a micro-switch datum. The detector module can be driven so that it completely contacts the cold face of the nitrogen can for maximum cooling power. When it is driven away from the cold face it is still thermally coupled to the can by copper braids.

The main blocking filter will be mounted on the detector module itself and light will not be able to get through to the detector except through this filter. It is $60 \times 60 \times 2 \mathrm{~mm}$. Two interchangeable detector modules will be made: one each for the HAWAII-I and HAWAII-II arrays. The one for the HAWAII-II array is offset so that only two quadrants get light from the camera optics. It is possible to rotate the array and its PCB so that the best two quadrants can be chosen.

Further filters can be accommodated in the two filter wheels, which locate filters between the third and fourth lenses of the camera. The large beam at this location requires filters of $170 \mathrm{~mm}$ diameter and the requirement that each wheel can take up to four such filters sets the diameter of the entire dewar. There are two filter wheels to give 5 filter options plus 2 clear and a blanked off position. It is not expected that we will use 2 wheel-mounted filters in the beam simultaneously. The filter wheels have teeth cut in to the perimeter so that they can be driven by a small spur gear driven by a stepper motor. Datum positions for the two wheels are provided by micro-switches and positioning is by step counting. 


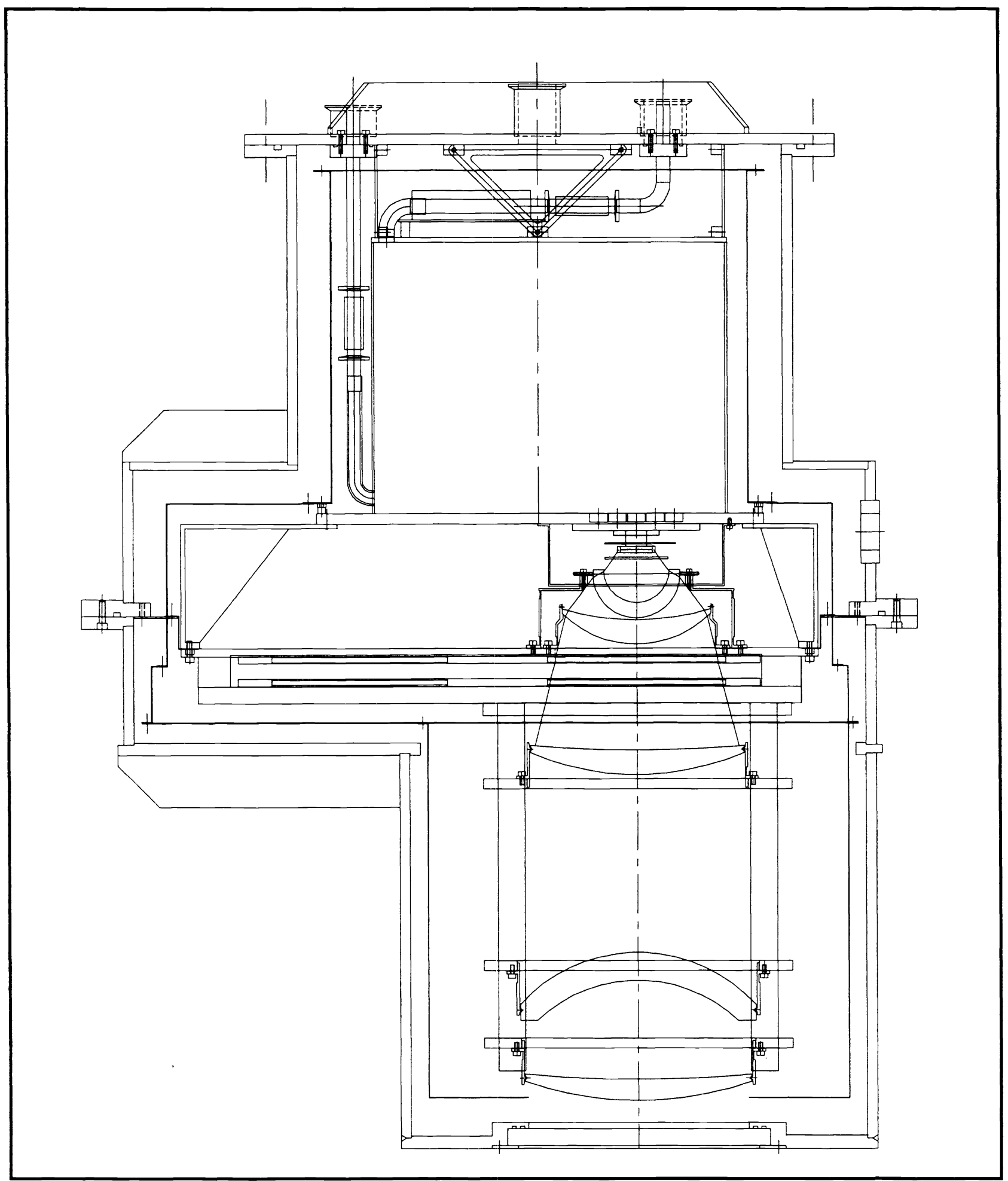

Figure 3. The cryogenic camera

The lens mounts are mounted in plates, which can be adjusted in position along the optical axis by sliding them along aluminium support tubes. The mounts can also be shimmed for de-centring and tilting. The first 3 lenses are mounted off the 
underside of the filter wheel and the last 2 lenses are mounted on the topside of the filter wheel. The entire filter-wheel plus lens assembly will be aligned at room temperature using one configuration of the "as built" Zemax model at optical wavelengths. The other configuration of this model is for IR wavelengths at $68 \mathrm{~K}$. The way one configuration relates to the other is modelled by Zemax to include the optical materials and the aluminium mounting structure. Therefore, once cooled the optics should be aligned optimally.

\subsection{The pros and cons of having a hardware suppression capability}

We have constructed CIRPASS using the front end of the COHSI spectrograph and we are therefore retaining the hardware $\mathrm{OH}$ suppression capability. This has the following advantages and disadvantages:

Advantages:

- The optics already exist and are known to give good performance

- Hardware removal of the $\mathrm{OH}$ at the mask will reduce the background due to scattering in the camera and in particular due to out-of-focus ghost images caused by the relatively reflective detector and filters.

- Persistent images due to strong $\mathrm{OH}$ lines will be eliminated and will therefore not cause problems when the data format on the detector is changed (e.g. to change wavelength range).

- The optical layout required to give an intermediate spectral image for masking incorporates a grating in a near-Littrow configuration that minimises losses due to anamorphism and allows large grating angles (giving high spectral resolutions).

- The optical layout for masking also includes a white pupil, which makes the design of a high throughput camera much simpler to achieve.

Disadvantages:

- The additional masking optics (the extra optics between the grating and the camera) have a throughput of $79 \%$.

- The overall size of the instrument is increased which is particularly undesirable, as the whole spectrograph has to be cooled.

The continuum flux from typical targets is likely to be fainter than the sky in between the $\mathrm{OH}$ lines which are in turn on average 30-50 times brighter. The total amount of $\mathrm{OH}$ flux collected by any instrument is proportion to the telescope aperture, the throughput, the FOV and the wavelength coverage. These are, of course, the parameters that all state-of-the-art NIR spectrographs attempt to maximise and CIRPASS is no exception. A straight-forward approach adopted by some $8 \mathrm{~m}$ spectrographs is to let the $\mathrm{OH}$ photons through to the detector and then ignore the small fraction of pixels they hit (i.e. socalled software suppression). CIRPASS can readily work in this mode too if the mask is replaced by a mirror

However some fraction of the $\mathrm{OH}$ flux will inevitably be scattered by the optics in the spectrograph (especially the optics near the detector) and be spread over all the detector pixels. The CIRPASS design will minimise the effects of scattering by allowing the option of hardware suppression to remove the $\mathrm{OH}$ as soon as possible in the optical train. This is a very important point for $\mathrm{J}$ and $\mathrm{H}$ band spectroscopy because the sky brightness in between the $\mathrm{OH}$ lines is so faint and the detector dark current is so low that the limiting background may well be due to scattered light if nothing is done to minimize it. The loss in throughput incurred by allowing this option is very small and the overall gain in signal-to-noise can be considerable.

The scattered light fraction in CIRPASS can be estimated to be as high as $\sim 2.5 \%$ simply by considering the reflections off the detector and the two sides of the blocking filter which is very close to it. It is reasonable to assume that the reflectivity of the detector is $25 \%$ and the reflectivity of the blocking filter is $5 \%$. OH light can reflect off the detector and then reflect back to the detector from the blocking filter. The amount of light on the detector in an out-of-focus ghost is therefore $0.25 \times 0.05=1.25 \%$ for one filter side and because there are 2 reflecting surfaces, $\sim 2.5 \%$ of the OH light could be smeared across the detector in the form of out-of-focus ghosts.

We have looked at the loss of sensitivity this scattering will give and the results are given in Table 1. The first column of data is for CIRPASS with an OH mask. The second column is for a theoretical spectrograph with no suppression capability and therefore higher throughput and higher scattering. The third column is for CIRPASS with the mask replaced by a plain mirror so the $\mathrm{OH}$ is not suppressed. The table shows that CIRPASS with suppression is as sensitive as the theoretical spectrograph. The assumptions made are that the intrinsic sky between the lines is $300 \mathrm{photons} / \mathrm{sec} / \mathrm{arcsec}^{2} / \mu \mathrm{m} / \mathrm{m}^{2}$, the 
instrumental background is $0.02 \mathrm{e} / \mathrm{sec} / \mathrm{pixel}$, the readnoise is $7 \mathrm{e} \mathrm{rms,} \mathrm{the} \mathrm{short} \mathrm{H}$ window is being observed, the object light is collected by a single 0.33 arcsec fibre and the total exposure time is 3 hours. The table therefore assumes that the interline sky brightness is very faint and we can keep the instrument background down to a very low level by cooling the detector, cooling the suppressor and using excellent filters.

Table 1. Photon arrival rates and the effects of scattering

\begin{tabular}{|l|c|c|c|}
\hline & $\begin{array}{c}\text { CIRPASS } \\
\text { with mask }\end{array}$ & $\begin{array}{c}\text { Theoretical spectrograph } \\
\text { with no suppression optics }\end{array}$ & $\begin{array}{c}\text { CIRPASS } \\
\text { without mask }\end{array}$ \\
\hline $\begin{array}{c}0 \% \text { scattering } \\
10.0 \% \text { throughput }\end{array}$ & $\begin{array}{c}2.5 \% \text { scattering } \\
12.7 \% \text { throughput }\end{array}$ & $\begin{array}{c}2.5 \% \text { scattering } \\
10.0 \% \text { throughput }\end{array}$ \\
\hline $\begin{array}{l}\text { Limiting mag in H } \\
\text { S/N=5 }\end{array}$ & 21.86 & 21.86 & 21.67 \\
\hline Scattered background $\ddagger$ & 0.0 & 0.035 & 0.028 \\
\hline Sky $\ddagger$ & 0.014 & 0.018 & 0.014 \\
\hline Object $\ddagger$ & 0.009 & 0.011 & 0.010 \\
\hline Brightest OH line $\ddagger$ & 0.25 & 68 & 54 \\
\hline
\end{tabular}

$\ddagger$ units are electrons/sec/pixel

It is important to note that there was no significant disadvantage or risk associated with keeping the hardware suppression/mask option. Only a marginal improvement in throughput is possible in principle by avoiding the extra two passes through the collimator and the mask itself. The 3 mirrors and 4 air-glass surfaces that could be removed have a measured total efficiency of $79 \%$. In practice these optics cannot be simply removed and we cannot conceive of a design that uses a conventional grating that would actually be $1 / 0.79 \times$ more efficient because alternative designs will have to deal with anamorphism, groove-shadowing and larger lights beams.

\subsection{Predicted Performance on Gemini}

Table 2 shows the limiting magnitude and limiting flux for a single fibre in the IFU for the various sampling scales we will offer on Gemini. The throughput (from outside the earth's atmosphere to recorded photons) is assumed to be $10 \%$, which we believe to be a realistic scenario. Flux limits are given in ergs $/ \mathrm{cm}^{2} / \mathrm{sec}$. The limits are for a S/N ratio of 5 and refer to a 2 pixel spectral resolution element. The following assumptions were made in predicting these performance limits. The total instrument dark current is $0.1 \mathrm{e} / \mathrm{sec} / \mathrm{pixel}$, which includes the scattered light, the thermal background and the intrinsic detector dark current. The array is reset every 30 minutes and the readnoise in a 30 minute exposure is $7 \mathrm{e}$ rms. The 0.05 arcsec/lens scale is when CIRPASS is used with Hokupa'a and an additional factor of $\times 0.8$ on throughput is assumed.

Table 2. $5 \sigma$ sensitivity limits for an exposure time of 3 hours and a throughput of $10 \%$

\begin{tabular}{|c|c|c|c|c|c|c|}
\hline $\begin{array}{c}\text { Spatial sampling } \\
(\operatorname{arcsec})\end{array}$ & $\begin{array}{c}\text { Spectral } \\
\text { resolution }\end{array}$ & $\begin{array}{c}\text { No. of object } \\
\text { fibres }\end{array}$ & $\begin{array}{c}\text { J flux limit } \\
\left(\mathrm{ergs} / \mathrm{cm}^{2} / \mathrm{s}\right)\end{array}$ & $\begin{array}{c}\mathrm{H} \text { flux limit } \\
\left(\mathrm{ergs} / \mathrm{cm}^{2} / \mathrm{s}\right)\end{array}$ & $\mathrm{J}_{\text {lim }}$ & $\mathrm{H}_{\text {lim }}$ \\
\hline 0.33 & $30.0 \AA \ddagger$ & 4 & $9.2 \times 10^{-18}$ & $6.7 \times 10^{-18}$ & 22.62 & 21.68 \\
\hline 0.33 & $4.4 \AA$ & 1 & $1.8 \times 10^{-18}$ & $1.3 \times 10^{-18}$ & 22.31 & 21.37 \\
\hline 0.25 & $4.4 \AA$ & 1 & $1.7 \times 10^{-18}$ & $1.3 \times 10^{-18}$ & 22.35 & 21.42 \\
\hline 0.12 & $4.4 \AA$ & 1 & $1.7 \times 10^{-18}$ & $1.2 \times 10^{-18}$ & 22.40 & 21.47 \\
\hline 0.05 & $4.4 \AA$ & 1 & $2.1 \times 10^{-18}$ & $1.5 \times 10^{-18}$ & 22.17 & 21.24 \\
\hline
\end{tabular}

$\ddagger$ resolution of data after rebinning from $4.4 \AA$ resolution

\section{INSTRUMENT COOLING}

To cool the entire spectrograph to $-40^{\circ} \mathrm{C}$ we have put it in a custom-made freezer chamber. The cooling is provided by a conventional single stage compressor-evaporator system using a standard refridgerant. The evaporator unit in the chamber has built in fans to cool and circulate the air. Before cooling, the chamber is purged of moisture by circulating the air through desicators. A slight over-pressure in the chamber ensures that moisture from the ambient air does not get in. 
Without this cooling the integrated thermal background in the $\mathrm{H}$ band is $\sim 140 \mathrm{e} / \mathrm{sec} / \mathrm{pixel}$ which is far too high in comparison to the other background sources. Even at $-40^{\circ} \mathrm{C}$ a filter blocking the background longward of $1.9 \mu \mathrm{m}$ is required. The chamber would have to be cooled to $-110^{\circ} \mathrm{C}$ to give a sufficiently low background without any blocking filters.

\section{INTEGRAL FIELD UNIT AND FORE-OPTICS}

Our IFU consists of a lens array that forms an array of micro-pupil images which are precisely aligned with an array of optical fibres. The array has 499 lenses and the geometry is shown in Figure 4. It is based very closely on the 100-element IFU that we built for COHSI ${ }^{5.6}$. Each lens measures $3 \mathrm{~mm}$ across the corners.

Table 3. Image scale options using the IFU

\begin{tabular}{|c|c|c|}
\hline $\begin{array}{c}\text { Lenslet Scale } \\
\text { (arcsecs/lenslet) }\end{array}$ & $\begin{array}{c}\text { Field size } \\
(\operatorname{arcsecs})\end{array}$ & Focal station \\
\hline 0.33 & $12.2 \times 5.0$ & F/16 \\
\hline 0.25 & $9.3 \times 3.5$ & F/16 \\
\hline 0.12 & $4.4 \times 1.7$ & F/16 \\
\hline 0.05 & $1.8 \times 0.7$ & F/20 Hokupa'a \\
\hline 7.0 & $259 \times 107$ & IoA 40cm Schmidt \\
\hline
\end{tabular}

The f-ratio at the Gemini focus is $\mathrm{f} / 16$ and we use a small lens to magnify the raw telescope image to the appropriate scale at the lens array. For the $\mathrm{f} / 16$ focus we provide 3 lens options to give 3 different scales. A fourth lens can be used with the f/20 focus of Hokupa'a and this provides a very finely sampled scale. These scale options and their corresponding fields of view are detailed in Table 3.

\section{MULTI-OBJECT MODE}

This will be implemented using a plug-plate system and a FOV of 6-7 arcmins will be provided. There will be 128 fibres and each one will have a small lens on the end to convert the $\mathrm{f} / 16$ beam of the telescope to $\sim \mathrm{f} / 6$ and ensure good matching to the f/5.5 collimator in the spectrograph. The closest any two fibres can be placed will be $\sim 14$ arcsec. These lenses will image the telescope pupil on to the end face of each fibre. The system will also incorporate special fibres for acquisition and guiding.

\section{DETECTORS}

We are currently operating CIRPASS with a $1024 \times 1024$ HAWAII-I array and we will upgrade to a $2048 \times 2048$ HAWAII-II array within a few months of receiving one from Rockwell. We hope to be one of the first instrument teams to operate a HAWAII-II on an $8 \mathrm{~m}$ telescope.

A commercial CCD controller with some interface electronics we developed in-house drives the array. The electronic scheme is similar to those for CIRSI, COHSI and UFTI. The CCD controller is controlled by a commercial software package called PIXCEL which we have modified specifically for IR array use.

\section{CONTROL SYSTEM AND COMPUTERS}

All mechanisms that need to be routinely adjusted during the night are controlled remotely. These include operation of the steering mirror, focus of the slit/mask assembly, focus and tilt of the detector, grating angle adjustment and choice of image scale on the IFU. Other operations such as changing masks and gratings require a manual change over. Special cryogenic stepper motors are used where required. The computers in the telescope control room are linked to the ones at the instrument using optical fibres. The control computers are PCs running Windows 98. A PC running Linux is used to run IRAF for data reduction. 


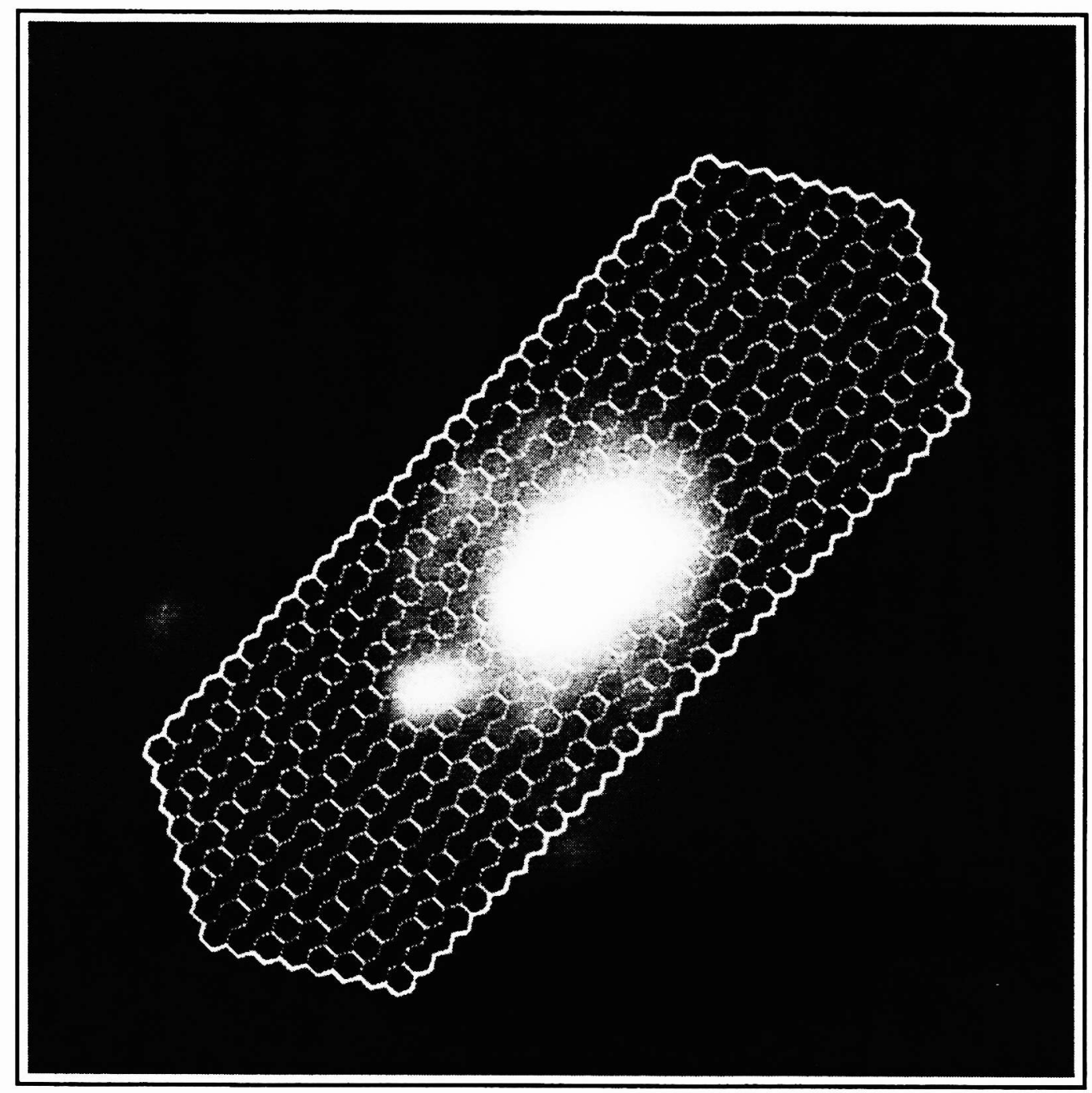

Figure 4. The arrangement of the 499 element lens-array in the IFU.

\section{CONCLUSIONS}

By upgrading an existing spectrograph (COHSI) we have been able to build an 8 -m class NIR spectrograph which will be a very powerful augmentation to the Gemini facility instrument suite. The CIRPASS project includes many state-of-the-art components including the Gemini telescopes, adaptive optics, a HAWAII-II array, an IFU, OH suppression masks, high optical density blocking filters and a new method of instrument cooling. By operating CIRPASS ourselves on behalf of the Gemini community we save time and money because CIRPASS does not have to conform to the rigid standards for facility instruments and there is no need for Gemini staff to learn how to operate the instrument.

\section{ACKNOWLEDGEMENTS}

We acknowledge the very valuable assistance of the Gemini Project, the ATC, PPARC and the Raymond and Beverly Sac' ler Foundation. 


\section{REFERENCES}

1. I. R. Parry, A. J. Dean, R. S. Ellis, D. King, C. D. Mackay, R. G. McMahon, S. R. Medlen, J. M. Pritchard, A. N. Ramaprakash, 2000, "CIRPASS - a NIR Integral Field Spectrograph", ASP conference series vol. 195, 191. "Imaging the Universe in Three Dimensions", eds. W van Bruegel and J Bland-Hawthorn.

2. Piché, F, Parry IR, Ennico, KA, Ellis, RS, Pritchard, JM, Mackay, CD, McMahon, RG, 1997, "COHSI: The Cambridge OH Suppression Instrument", Proc of SPIE vol 2871, 1332.

3. Ennico, KA, Parry, IR, Kenworthy, MA, Ellis, RS, Mackay, CD, Beckett, MG, Aragón-Salamanca, A, Glazebrook, K, Brinchmann, J, Pritchard, JM, Medlen, SR, Piché, F, McMahon, RG, Cortecchia, F, 1998, "The Cambridge OH Suppression Instrument (COHSI): Status After First Commissioning Run", Proc of SPIE, 3354, 668.

4. Graves, J, Northcott, MJ, Potter, D, O'Connor, DJ, Roddier, CA, Roddier, FJ, Figaut, FJ, Chun, MR, 2000, Proc. Of SPIE Vol. 4007, "First light for Hokupa'a 36 on Gemini North".

5. Kenworthy, MA , Parry, IR, Ennico KA, 1998 "COHSI: a lens array and fibre feed for the near infra-red", proc. of the conference "Fibre Optics in Astronomy - III", ASP conf. series vol 152, 300, eds. Arribas, Mediavilla and Watson.

6. Kenworthy, MA, Parry, IR, Taylor, K, 1998, "Integral Field Units for SPIRAL and COHSI", Proc. SPIE Vol. 3355, p. 926-931, Optical Astronomical Instrumentation, Sandro D'Odorico; Ed. 